\title{
Laparoscopic radical prostatectomy: continence and oncological outcomes in 268 cases
}

\author{
S. S. Goonewardene $e^{1,2} \cdot$ D. Cahill ${ }^{3}$
}

Received: 26 June 2015/ Accepted: 12 July 2015 / Published online: 26 July 2015

(C) Springer-Verlag London 2015

\section{Dear Sir,}

We were delighted to read the article by Palisaar et al. Urinary incontinence is a very important topic post-open or robotic prostatectomy. However, we believe it is also important to consider results post-laparoscopic prostatectomy. There is little laparoscopic radical prostatectomy data to benchmark realistic outcomes to inform patient treatment choice.

A prospective database of 268 laparoscopic prostatectomies (single centre, single surgeon, 2003-2009) was reviewed for age, stage and grade, continence, clinical recurrence time, positive margins, metastases and death.

The mean age was 60.2 years (range 36-73). The average time to continence (no leakage and no pads) was 4.5 months if below 70 years. Seven cases required an artificial urinary sphincter, 3 required male slings, 4 were injected with bulking agents, 2 required botox injections (6\% requiring continence interventions), 3 required urethral dilatations.

There were nine patients $>70$ years. All patients were alive, with no deaths or metastases. The average time to continence was 5.7 months. One patient $(11 \%)$ required injection of bulking agent, another urethral dilation.
This was re-iterated by [1, 2], who demonstrated functional and oncological outcomes for laparoscopic prostatectomy are acceptable. This clearly demonstrates the role of laparoscopic surgery in maintaining good urinary continence. Patients need realistic data to make choices. Radical prostatectomy is a good cancer treatment. Incontinence is a real long-term issue especially in the learning curve.

\section{Compliance with ethical standards}

Conflict of interest S.S. Goonewardene and D. Cahill declare that they have no conflict of interest.

\section{References}

1. Verze P, Scuzzarella S, Martina GR et al (2013) Long-term oncological and functional results of extraperitoneal laparoscopic radical prostatectomy: one surgical team's experience on 1,600 consecutive cases. World J Urol 31(3):529-534. doi:10.1007/ s00345-013-1052-0

2. Goeman L, Salomon L, De Taille AL et al (2006) Long-term functional and oncological results after retroperitoneal laparoscopic prostatectomy according to a prospective evaluation of 550 patients. World J Urol 24(3):281-288. doi:10.1007/s00345-0060054-6
S. S. Goonewardene

ssg7727@yahoo.co.uk

Guys and St Thomas Hospitals, London, UK

2 Great Western Hospitals, Swindon, UK

3 The Royal Marsden Hospital, London, UK 